\title{
A histopathological analysis of the human cervical spinal cord in patients with acute traumatic central cord syndrome
}

\author{
O Jimenez ${ }^{1}$, A Marcillo $^{1}$ and ADO Levi*,1 \\ ${ }^{1}$ The Miami Project to Cure Paralysis, Department of Neurosurgery, Jackson Memorial Hospital, University of \\ Miami, Florida, USA
}

\begin{abstract}
Study design: We have applied conventional histochemical and morphometric techniques to study the changes within the human spinal 'hand' motor neuron pool after spinal cord injury in patients who presented with acute traumatic central cord syndrome (ATCCS).

Objective: To determine whether a reduction of large alpha motor neurons at the C7, C8 and T1 spinal cord levels underlies the mechanism which causes hand dysfunction seen in patients with (ATCCS).

Background: The etiology of upper extremity weakness in ATCCS is debated and injury and/ or degeneration of motor neurons within the central gray matter of the cervical enlargement has been advanced as one potential etiology of hand weakness.

Methods: The spinal cords of five individuals with documented clinical evidence of ATCCS and three age-matched controls were obtained. The ATCCS spinal cords were divided into acute/sub-acute (two cases) and chronic (three cases) groups depending on the time to death after their injury; the chronic group was further subdivided according to the epicenter of injury. We counted the motor neurons using light microscopy in 10 randomly selected axial sections at the C7, C8 and T1 spinal cord levels for each group. We also analyzed the lateral and ventral corticospinal tracts (CST) in all groups for evidence of Wallerian degeneration and compared them to controls.

Results: A primary injury to the lateral CST was present in each case of ATCCS with evidence of Wallerian degeneration distal to the epicenter of injury. There was minimal Wallerian degeneration within the ventral corticospinal tracts. In the chronic low cervical injury group, there was a decrease in motor neurons supplying hand musculature relative to the other injury groups where the motor neurons sampled at the time of death were not reduced in number when compared to the control group.

Conclusions: We hypothesize that hand dysfunction in ATCCS can be observed after spinal cord injury without any apparent loss in the number of motor neurons supplying the hand musculature as seen in our acute/sub-acute $(n=2)$ and our chronic high injury $(n=1)$ groups. The motor neuron loss seen in the chronic low level injury was felt to be secondary to the loss of $\mathrm{C} 7, \mathrm{C} 8$, and $\mathrm{T} 1$ neurons adjacent to the injury epicenter.
\end{abstract}

Spinal Cord (2000) 38, $532-537$

Keywords: central cord syndrome; motor neurons; morphometry

\section{Introduction}

The etiology of upper extremity weakness in acute traumatic central cord syndrome (ATCCS) is debated. ${ }^{1-3}$ The differing viewpoints are at least partially based on whether any somatotopic organization exists within the human corticospinal tract (CST). The arguments for such an organization to this tract have very early origins within the neuroscience literature. Schneider ${ }^{4}$ described the syndrome of ATCCS combining several clinical cases and only one neuropathologic

*Correspondence: ADO Levi, The Miami Project to Cure Paralysis, University of Miami, 1501 N.W. 9th Ave., Suite 2011, Miami, Florida 33136, USA case. He felt that the syndrome resulted after the formation of a traumatic central hematomyelia damaging the most medially, located 'hand' CST fibers within the lateral columns. An article by Foerster (1937) was cited as a reference for CST organization within the lateral columns. In his paper, CST fibers controlling hand and arm movement were located medially, and leg fibers were positioned laterally within the tract.

A very similar clinical presentation consisting of disproportionate hand and arm weakness can be seen after high cervical cord injuries. In the 1970 s, Bell ${ }^{5}$ described the syndrome of cruciate paralysis (BCP) in 
'which both arms are paralyzed without weakness of the lower extremities.' Using the initial postulates of Wallenberg, ${ }^{6}$ he further hypothesized that an injury to the rostral aspects of the pyramidal decussation affecting the rostrally located hand and arm fibers of the CST and sparing the more caudally located leg fibers resulted in BCP. Somatotopic organization of the CST, however, has never been demonstrated in the spinal cord of humans or higher mammals to date, using either Marchi degeneration or anterograde tracing studies. ${ }^{1,2,7}$

Several experimental and neuropathologic observations have led us to suggest an alternative hypothesis to explain the relatively greater hand dysfunction seen with some incomplete cervical spinal cord injuries (ATCCS and BCP). ${ }^{1}$ Sectioning of the CST in monkeys results in relatively more upper extremity dysfunction relative to the lower extremities, ${ }^{8-12}$ and in particular fine finger movements are the most impaired. The CST has assumed relatively greater importance in new world monkeys with good manual dexterity and in humans where large axons of the CST tract synapse directly on alpha motor neurons which supply hand musculature. ${ }^{13-17}$ Bunge et al $^{14}$ demonstrated that, in patients with central cord syndrome, there is selective injury to the large corticospinal axons that synapse directly on hand motor neurons.

Alternative explanations ${ }^{1}$ advanced to explain the above syndromes include: (1) injury to central gray matter of the cervical enlargement at the level of the motor neurons supplying the hand muscles; (2) selective injury to the uncrossed ventral CST; and (3) selective injury to the forelimb CST collaterals to the brainstem, central gray and dorsal column nuclei. The current study characterizes histologically the motor neurons supplying hand musculature (C7T1) as well as the CST in postmortem human cervical spinal cord specimens in patients who presented with either BCP or ATCCS. We determined whether a reduction in motor neurons supplying hand muscles is observed in an attempt to elucidate the pathophysiologic mechanism for disproportionate upper extremity weakness seen in these syndromes.

\section{Materials and methods}

Cervical spinal cords of five patients diagnosed with either ATCCS or BCP were examined pathologically and compared to three postmortem age-matched controls. The cause of death in the patients with spinal cord injuries was due to respiratory or other medical complications related to their traumatic injury. The spinal cords were divided into an acute/sub-acute $(n=2)$ or a chronic group $(n=3)$ depending on the time of death after the spinal cord injury. The acute/subacute group died within 5 weeks of injury, while the chronic group died more than 5 weeks after injury. The level of injury for the acute/sub-acute group was at C5 $(n=2)$. The chronic group was further sub-divided into a high level injury group $(\mathrm{C} 2 ; n=1)$ and a low level injury group at $\mathrm{C} 5-\mathrm{C} 7(n=2)$.

The age-matched control spinal cord specimens were obtained from three patients ranging in age from 73-77 years old. Two patients died from coronary artery disease and the other from rupture of an intracerebral artery aneurysm. The human spinal cords were harvested $12-24 \mathrm{~h}$ postmortem and fixed immediately in $10 \%$ buffered formalin solution. The spinal cords remained in fixative for at least 2 weeks prior to sectioning. Each cervical level was embedded in paraffin and $6-\mu \mathrm{m}$ sections were obtained using a microtome. The sections were then stained with hematoxylin-eosin $(\mathrm{H} \& \mathrm{E})$ for motor neuron counts or a Sevier-Munger (SM) stain for an analysis of tract anatomy within the lateral columns.

Ten axial sections were selected randomly from the C7, C8, and T1 spinal cord levels for all groups. In the patient with the chronic high level (C2) injury, we only counted seven sections at $\mathrm{C} 7$ due to poor tissue preservation at this level. The remainder of the levels (C8 and T1) in this patient were subjected to the above described analysis. The alpha motor neurons were counted using the morphology criteria reported by Tomlinson et al. ${ }^{18}$ A micrometer was used to measure the motor neuron maximum diameter; motor neurons identified within the medial or lateral ventral horn measuring greater than $30 \mu \mathrm{m}$ in diameter was included in the counts. Histological analysis of the ventral and lateral CST was performed at the site of injury as well as rostral and caudal to the level of injury.

\section{Results}

\section{Clinical histories and imaging}

Acute/sub-acute I A 67-year-old male fell off the toilet and hit his forehead on the floor and was identified 2 days later. He had grade $3 / 5$ strength of his proximal upper extremities. He presented with grade $0 / 5$ strength in his distal upper extremities. His strength in his lower extremities $24 \mathrm{~h}$ post-admission was grade $1-2 / 5$. The patient had a history of alcohol abuse, a seizure disorder and previous evacuation of an acute subdural hematoma. His CT and MRI scan (Figure 1A) of his cervical spine revealed diffuse spondylosis of $\mathrm{C} 3$ to $\mathrm{C} 7$ with the most severe spinal stenosis at $\mathrm{C} 3-$ $\mathrm{C} 4$ and less so at the $\mathrm{C} 6-\mathrm{C} 7$ level. No fractures were identified. He died from sudden respiratory failure and hypotension secondary to a pulmonary embolus 3 days post injury.

Acute/sub-acute II An 80-year-old male pedestrian was hit by a motor vehicle. He presented with grade $0 /$ 5 strength in his upper extremities and $1-2 / 5$ in the lowers. Plain X-rays and a CT scan of the cervical spine revealed mid-cervical stenosis secondary to posteriorly projecting osteophytes worse at the C3- 

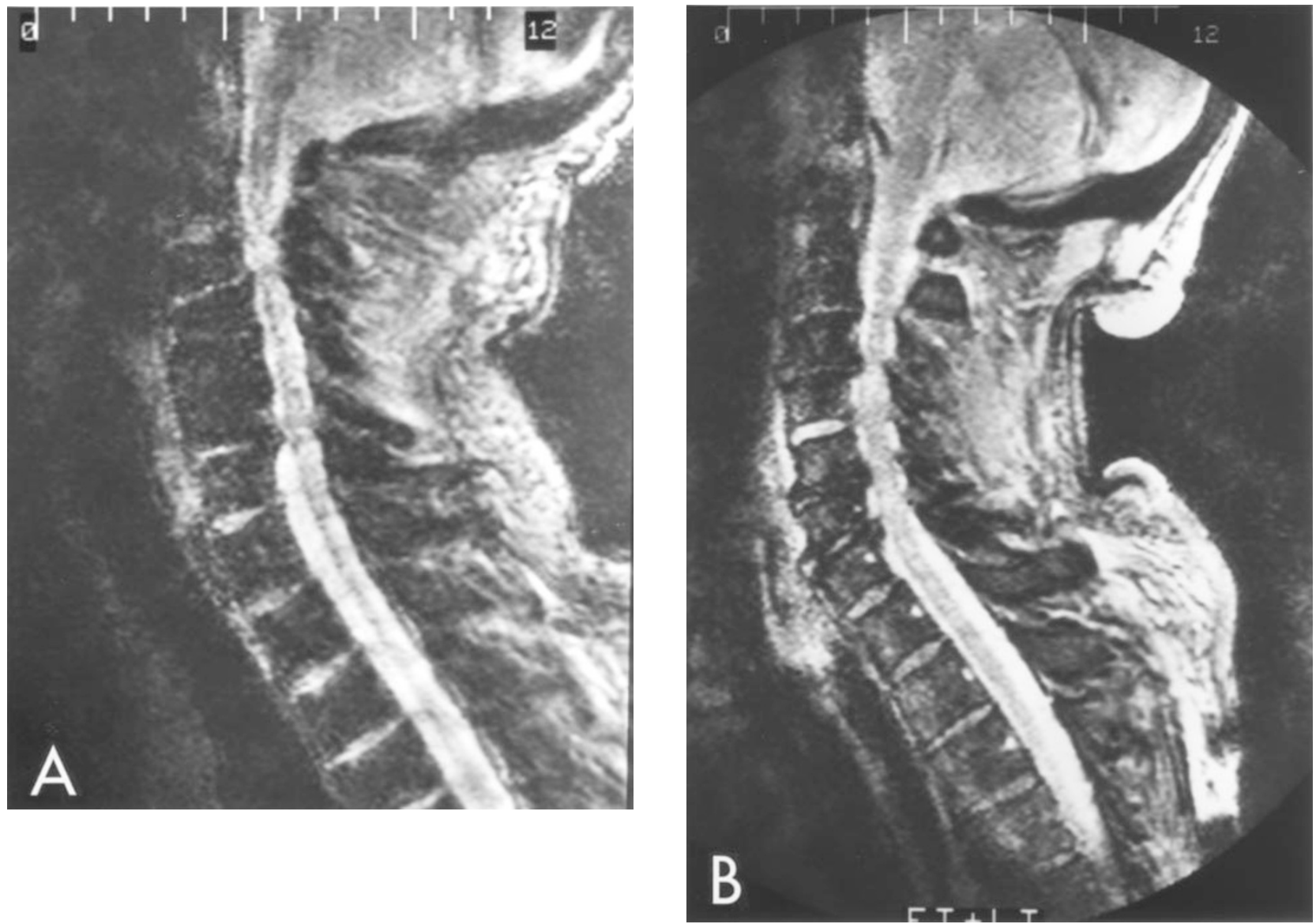

Figure 1 (A) Sagittal T2-weighted MRI of acute/sub-acute case I demonstrating multi-level cervical spinal stenosis from C3 to C7 with the most severe spinal stenosis at C3C4 and less so at the C6C7 level. (B) Sagittal T2-weighted MRI scan demonstrated significant spinal stenosis and cord compression over secondary to posteriorly projecting osteophytes and ligamentum hypertrophy worse at the $\mathrm{C} 3 \mathrm{C} 4$ and $\mathrm{C} 5 \mathrm{C} 6$ levels

C4 and C5-C6 levels. No fractures were identified. The MRI scan demonstrated significant spinal stenosis and cord compression over these same levels (Figure 1B). Respiratory failure, renal failure and arrhythmias complicated his hospital course. He ultimately died from complications related to sepsis 34 days postinjury.

Chronic I- high An 86-year-old male fell from a deer stand. He was found the following day by his companions. He presented with grade $0 / 5$ strength in the upper extremities and $3 / 5$ strength in the lower extremities. Plain X-rays demonstrated a type II odontoid fracture $(\mathrm{C} 2)$. He underwent a $\mathrm{C} 1 \mathrm{C} 2$ Brooks fusion with iliac crest autograft and methylmethacrylate. He recovered ambulatory function with a walker and some proximal upper extremity strength but was unable to groom or feed himself. He died from respiratory complications 11 months post-injury.

Chronic II-low A 78-year-old male was injured in an automobile accident. The patient presented with weakness in his hands, which was more pronounced, than in the arms and legs. Pre-mortem magnetic resonance imaging (MRI) and plain X-rays revealed severe spinal stenosis with compression of the thecal sac and the cervical cord at the $\mathrm{C} 3-\mathrm{C} 4$ level, with milder stenosis at $\mathrm{C} 4-\mathrm{C} 6$ levels. There was no evidence of fracture subluxation.

Coronal and sagittal post-mortem MRI revealed hyperintense signals at $\mathrm{C} 5$ and rostral and caudal to the compressed region of the cord. The rostral extension of the abnormal signal was limited to approximately one centimeter, while the caudal extension reached for $3-4 \mathrm{~cm}$ below the C5 epicenter of the compression. There was no MRI evidence of damage in the upper cervical, lower thoracic, lumbar and sacral levels of the cord. He survived for 3.5 months and then died of pneumonia.

Chronic III - low A 64-year-old male was involved in a motor vehicle accident. He presented with upper extremity weakness (grade $3 / 5$ proximally and $1 / 5$ distally). In his lower extremities he had $4 / 5$ strength. Plain X-rays and CT scan of the cervical spine revealed a $\mathrm{C} 6-\mathrm{C} 7$ fracture involving the left lamina with 
disruption of the $\mathrm{C} 5-6$ and $\mathrm{C} 6-7$ facet joints. $\mathrm{He}$ underwent a C5-T1 laminectomy. His hospital course was complicated by a wound infection 21 days after surgery. Twelve days later he underwent a C6-C7 anterior exploration for discitis and repair of a CSF leak. Fifty days post-admission he was found to have an esophageal sinus tract. At 4 months post-injury he was transferred to our institution with a pseudomonas posterior cervical wound infection which required incision and drainage and placement of a Halo vest for instability. Five months post-injury he was found to be lethargic, febrile with hydrocephalus. CSF cultures grew yeast. He died approximately 6 months from the time of his initial injury from yeast meningitis.

\section{Histopathology}

The average age of the patients $(n=5)$ with ATCCS was 70.5 years while the age of the control group spinal cords $(n=3)$ was 75 years. The ATCCS spinal cords were divided into acute/sub-acute (two cases) and chronic (three cases) groups depending on the time to death after their injury; the chronic group was further subdivided according to the epicenter of injury. The average motor neuron counts for the acute/sub-acute $(n=2)$; chronic high $(n=1)$ and low $(n=2)$; and control groups $(n=3)$ are shown in Figure 2. The epicenter of injury pathologically for the acute/ sub-acute group was at C6 and C7 (two cases). The pathological epicenter of injury for the chronic high group was C2 (one case) and for the chronic low cervical injury group at $\mathrm{C} 5$ but the area of injury extended well inferiorly and $\mathrm{C} 6-\mathrm{C} 7$ (two cases). The average sum total number of motor neurons from C7-T1 for the control group was 1908; for the acute/ sub-acute group 1917; for the chronic high cervical level group 1877; and for the chronic low cervical level group 1168. There was an average of 733 less motor neurons in the chronic low cervical injury group when compared to the other groups. The average difference between the acute/sub-acute group and chronic high level group from the control was 20 motor neurons. Large motor neurons meeting the criteria described in the methods were found in the ventral-medial and lateral horns and were included (Figure 3). There was some variability in the size of the motor neurons as well as the shape of these cells (Figure 4). Motor neurons in the chronic low injury group were fewer in number, and of those that remained, some were fragmented. The motor neuron

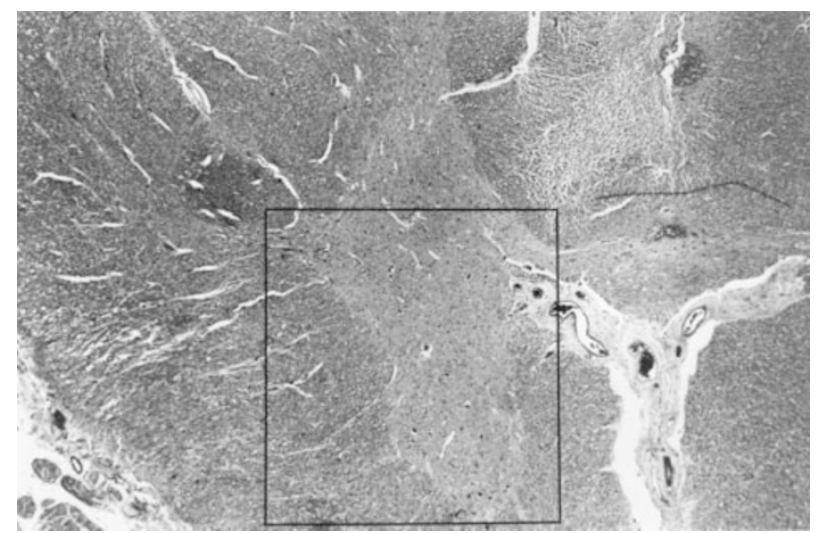

Figure 3 Location of large motor neurons in the ventral gray spinal cord. Spinal cord section at $\mathrm{C} 7$ from the control group. Large motor neurons found in the ventral medial and lateral aspect of each section were included in the motor neuron counts. Neurons at these locations for the C7, C8 and TI segments are known to control hand motor function in humans. Magnification $10 \times$

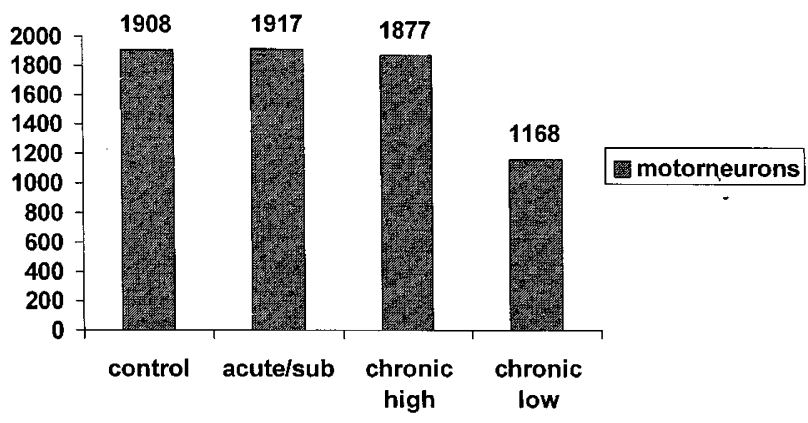

Figure 2 Average number of motor neurons at C7, C8 and T1 per injury type. Diagrammatic representation of the average total number of motor neurons per cervical spinal cord segment counted at C7, C8 and T1 versus injury type/ level. There was a minimal difference in the total number of motor neurons counted between the control spinal cords (1908), $n=3$; the high level injury group (1877), $n=1$; and the acute/sub-acute group (1917), $n=2$. The low level injury group (1168), $n=2$ had the fewest motor neurons when compared to the control and other injury groups

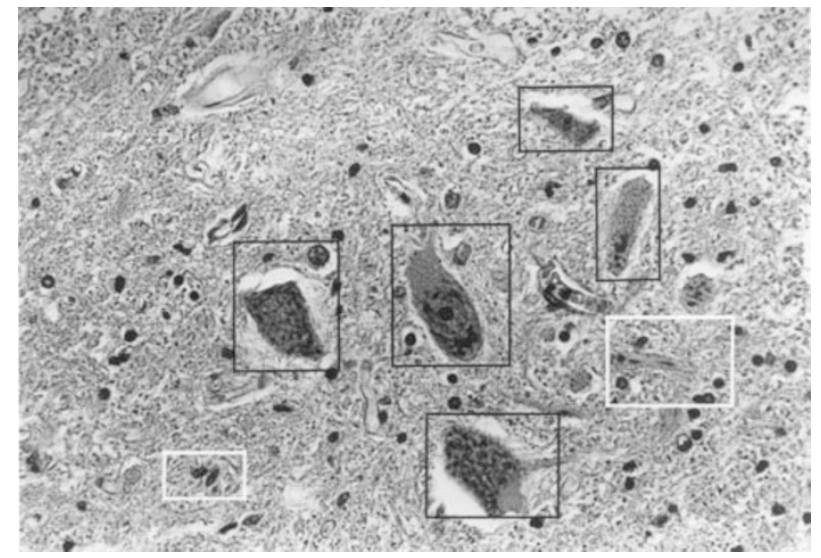

Figure 4 Selection criteria for counting motor neurons. Motor neurons were counted using a micrometer at $20 \times$ magnification. Large neurons measuring $30-70 \mu \mathrm{m}$ were included in the counts (black rectangles). Motor neurons less than $30 \mu \mathrm{m}$ in size were not counted (white rectangles) 


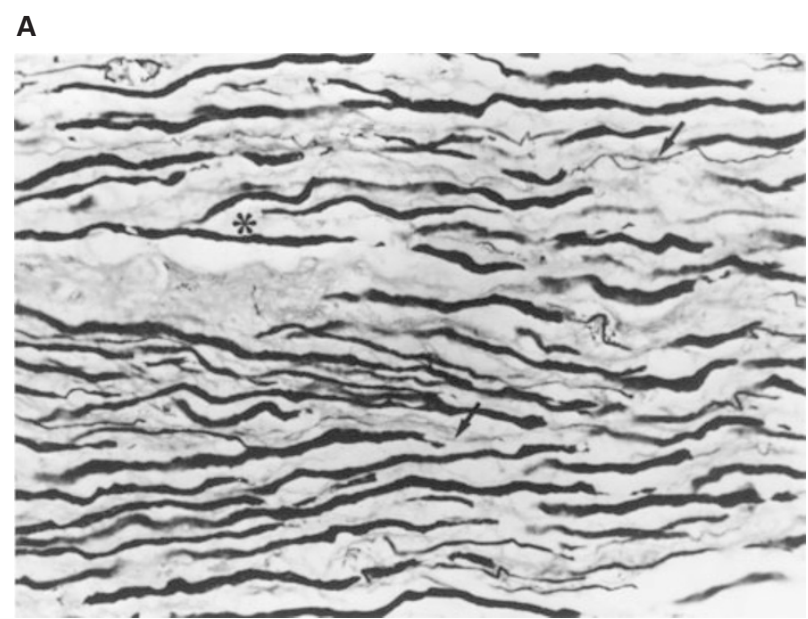

B

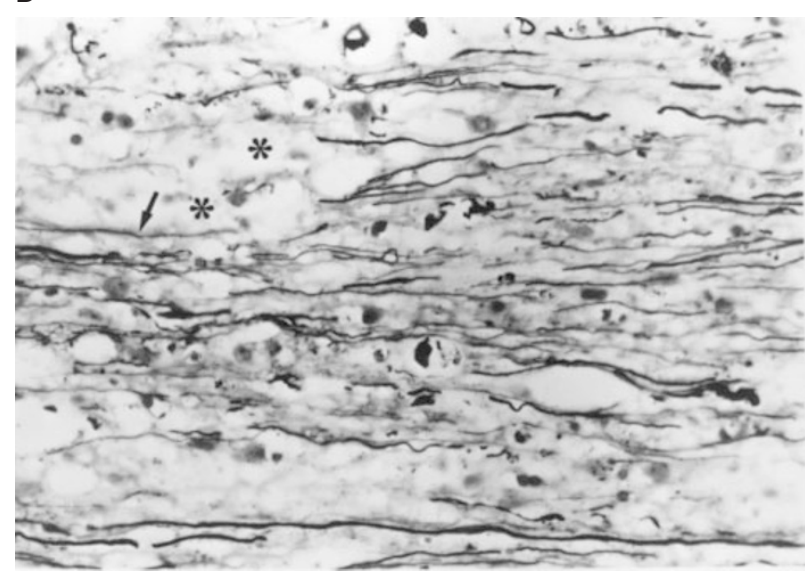

C

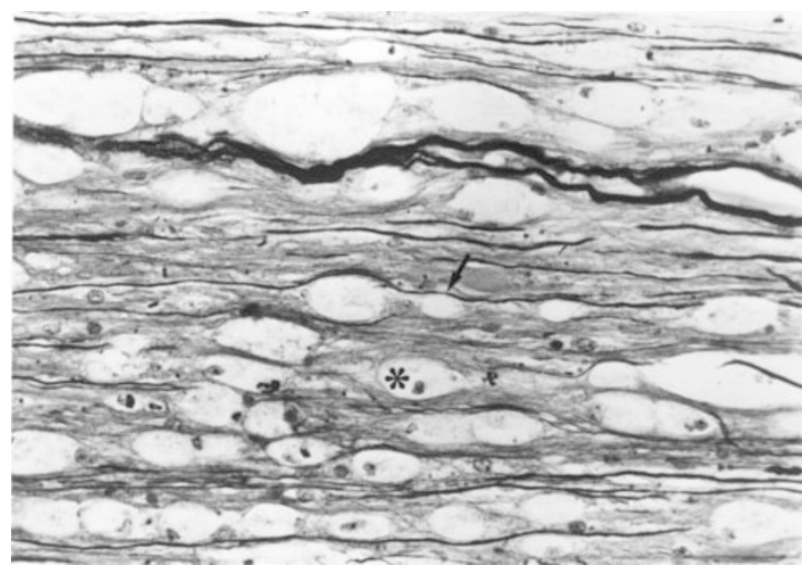

Figure 5 Wallerian degeneration in the lateral CST. Specimens were obtained as $6 \mu \mathrm{m}$ longitudinal sections through the lateral columns and prepared with a Sevier-Munger stain. All specimens were examined under $40 \times$ magnification. (A) CST fibers with the lateral columns of a control spinal cord. Note the preservation of the CST axons in this specimen with no history of spinal cord injury. Asterisk: Large myelinated CST fibers; arrow: small myelinated CST fibers. (B) Chronic low level injury group. The epicenter of injury for this specimen was at $\mathrm{C} 5$. Note the Wallerian degeneration and fragmentation of the myelin around large CST fibers at C6 (asterisk). pools in the chronic low injury group were nearer to the injury epicenter.

Degeneration of CST axonal tracts within the lateral columns was seen in each of the cases of ATCCS. The site of CST Wallerian degeneration was distal to the epicenter of injury. There was myelin fragmentation and loss of the larger CST fibers with some preservation of the smaller CST fibers in the chronic high and low level injury groups (Figure 5AC). For the acute/sub-acute groups there appeared to be preservation of the small and larger caliber CST, however, there was evidence of morphological enlargement of these fibers as well as the interstitial and intercellular space most consistent with traumatic edema.

We also examined axial sections of the ventral medial white matter tracts from $\mathrm{C} 7-\mathrm{T} 1$ within areas closely approximating the ventral CST fibers at $10 \times$, $40 \times$, and $100 \times$ for evidence of Wallerian degeneration (data not shown). There was very mild fragmentation of myelin in the chronic low and high injury group in contradistinction to the severe myelin fragmentation seen in the lateral columns.

\section{Discussion}

There have been few reports on the fate of motor neurons in chronic spinal cord injury. McComas, ${ }^{19-21}$ Serratrice, $^{22}$ and Young ${ }^{23,24}$ have demonstrated an electrophysiological dysfunction of motor units in muscles below a spinal cord injury or after cerebral infarction. Transynaptic degeneration of motor neurons secondary to injury of descending tracts with a loss of input upon these neurons was felt to be an important pathophysiologic mechanism. Stein ${ }^{25}$ and Taylor $^{26}$ have shown motor neuron loss in one patient with chronic high cervical spinal cord compression resulting in hand dysfunction. Using strict morphological criteria, a detailed analysis of motor neurons in traumatic spinal cord injury by Kaelan et $a l^{27}$ demonstrated the opposite result. These authors carefully counted motor neurons within the lumbosacral enlargement after chronic and pathologically complete thoracic or cervical spinal cord injuries and did not demonstrate any loss of motor neurons. These findings are in accordance with those of Oppenheimer ${ }^{28}$ and Khoubessarian ${ }^{29}$ who have shown no loss of motor neurons in humans with thoracic and lumbar CST lesions.

It is known that the large corticospinal axons have direct primary input into alpha motor neurons

There is some preservation of the thinner CST fibers (arrow). (C) Chronic high level injury group. The epicenter of injury for this specimen was at $\mathrm{C} 2$. Note the evidence of Wallerian degeneration and fragmentation of myelin in the CST at C8. Again notice the fragmentation of the large CST fibers (asterisk) and relative preservation of most of the smaller caliber CST fibers (arrows) 
supplying hand musculature from $\mathrm{C} 7, \mathrm{C} 8$ and $\mathrm{T} 1 .{ }^{13} \mathrm{It}$ is postulated that injury to these large CST axons may form the basis for hand weakness seen in ATCCS. ${ }^{1,3}$ In the current study, we have analyzed morphometrically the number of motor neurons at these levels to determine whether injury to the CST, which has been previously documented after ATCCS, ${ }^{1}$ results in motor neuron loss in cervical spinal cord segments which supply hand musculature. In the chronic high level injury $(\mathrm{C} 2)$, the epicenter of injury was furthest away from the motor neurons supplying hand muscles. In this case there was no motor neuron loss attributable to the traumatic injury, and the number of motor neurons was similar to the controls.

Similar findings were demonstrated in the acute/subacute group where there was no loss of motor neurons supplying hand musculature relative to the controls even though the epicenter of injury was relatively closer to hand motor neurons on pathological examination. This is in contrast to what was observed in the chronic low cervical injury group; where there was a decrease in the number of motor neurons when compared to the controls of the other injured groups. We postulate that in the chronic low injury group, the epicenter of injury was closer to the $\mathrm{C} 7, \mathrm{C} 8$ and $\mathrm{T} 1$ levels and thus destroyed a large number of these motor neurons, resulting in a lower total count. In the acute/sub-acute group with a C7 epicenter of injury, motor neuron degeneration had not occurred to an extent at the time of death ( 3 days to 5 weeks) which could be detected by simply counting motor neurons. We presume that if time to death after the traumatic injury was similar to the chronic low injury group, that the loss of motor neurons may have been greater. Delayed neuronal death due to apoptosis ${ }^{30}$ may conceivably be one mechanism to explain the delayed loss of motor neurons.

\section{Conclusions}

We propose that the hand dysfunction in acute traumatic central cord syndrome results from a primary injury involving the large fibers of the lateral CST and can occur in the absence of motor neuron loss supplying hand musculature. A reduction of the motor neuron pool supplying the hands was only seen in the chronic low cervical injury group where the epicenter of injury was adjacent to this area.

\section{References}

1 Levi ADO, Tator $\mathrm{CH}$, Bunge RP. Clinical syndromes associated with dysproportionate weakness of the upper versus the lower extremities after cervical spinal cord injury. Neurosurgery 1996; 38: $79-186$.

2 Pappas CT, Gibson AR, Sonntag VKH. Decussation of hindlimb and forelimb fibers in the monkey corticospinal tract: Relevance to cruciate paralysis. $J$ Neurosurg 1991; 75: $935-940$.

3 Quencer RM et al. Acute traumatic central cord syndrome: MRI pathological correlations. Neuroradiology 1992; 34: 85-94.
4 Schneider RC, Cherry G, Pantek H. The syndrome of acute central spinal cord injury. J Neurosurg 1954; 11: 546-577.

5 Bell HS. Paralysis of both arms from injury of the upper portion of the pyramidal decussation: 'Cruciate paralysis.' J Neurosurg 1970; 33: $376-380$.

6 Wallenberg A. Anatomischer Befund in einem als 'acute Bulbarraffection (embolie der Art cerebellar post. Inf. Sinsistr?)' beschrieben Falle. Arch Psychiatry 1901; 34: 923-959.

7 Coxe WS, Landau WM. Patterns of Marchi degeneration in the monkey pyramidal tract following small discrete cortical lesions. Neurology 1970; 20: $89-100$.

8 Bucy PC. Is there a pyramidal tract? Brain 1957; 80: 376-392.

9 Bucy PC, Keplinger JE, Siquiera EB. Destruction of the pyramidal tract in man. J Neurosurg 1964; 21: 385-398.

10 Bucy PC, Landpli R, Ehrlich A. Destruction of the pyramid tract in the monkey: The effects of bilateral section of the cerebral peduncles. J Neurosurg 1966; 25: 1-33.

11 Lawrence DG, Kuypers HGJM. The functional organization of the motor system in the monkey: I-The effects of bilateral pyramidal lesions. Brain 1968; 91: 1-14.

12 Lawrence DG, Kuypers HGJM. The functional organization of the motor system in the monkey: II-The effects of lesions of the descending brain stem pathways. Brain 1968; 91: 15-36.

13 Bortoff GA, Strick P. Corticospinal terminations in two new world primates. J Neurosci 1993; 13: 5105-5118.

14 Bunge RP et al. Observations on the pathology of human spinal cord injury. In: Seil FJ (ed). Advances in Neurology. Raven Press: New York, 1993, pp 75-89.

15 Hefner R, Masterson B. Variation in the form of the pyramidal tract and its relationship to digital dexterity. Brain Behav Evol 1975; 12: $161-200$.

16 Kuypers HGJM. Anatomy of the descending pathways. In: Brookhart JM, Mountcastle VB (eds). Handbook of Physiology: The nervous system. Vol. 2. Williams \& Wilkins: Baltimore, 1981, pp 597-647.

17 Phillips CG, Porter R. Corticospinal neurons: Their role in movement. In: Monographs of the Physiological Society. Vol. 34 Academic Press: London, 1977, pp 1-19 and 357-368.

18 Tomlinson BE, Irving D, Rebeiz JJ. Total numbers of limb motor neurones in the human lumbosacral spinal cord and an analysis of the accuracy of various sampling procedures. J Neurol Sci 1973; 20: $313-327$.

19 McComas AJ, Sicca RE, Upton ARM, Aguilera N. Functional changes in motorneurons in hemiparetic changes. $J$ Neurol Neurosurg Psychiat 1973; 36: 183-193.

20 McComas AJ, Sicca RE, Campbell MJ. 'Sick' motorneurons: a unifying concept of muscle disease. Lancet 1971b; I: 321-325.

21 McComas AJ et al. Motorneuron dysfunction in patients with hemiplegic atrophy. Nat New Biol 1971a; 233: 21-23.

22 Serratrice G et al. Amyotrophies et depopulations neuronale d'origine encephalique. Rev Neurol 1975; 131: 185-192.

23 Young JL. Morphological and histochemical studies of partially and totally deafferented spinal cord segments. Exp Neurol 1966; 14: $238-248$

24 Young JL, Mayer RF. Physiological alterations of motor units in hemiplegia. J Neurol Sci 1982; 54: $401-412$.

25 Stein BM, Leeds NE, Taveras JM, Pool JL. Meningiomas of the foramen magnum. Neurosurgery 1963; 20: $740-751$.

26 Taylor AR, Byrnes DP. Foramen magnum and high cervical cord compression. Brain 1974; 97: 473-480.

27 Kaelan C, Jacobsen PF, Kakulas BA. An investigation of the possible transynaptic neuronal degeneration in human spinal cord injury. J Neurol Sci 1988; 86: $231-237$.

28 Oppenheimer DR. Diseases of the basal covelia, cerebellum and motor neurons. In: Hume-Adams J, Corsellis JAN, Duchen LW (eds). Greenfield's Neuropathology. 4th edn. Edward Arnold: London, 1984, pp 725-726.

29 Khoubesserian P, Telerman-Toppet N, Welraeds D, Coers C. Modifications des unites motricses chez des malades atteints de paraplegie traumatique. Rev Neurol 1983; 139: 635-642.

30 Crowe $\mathrm{M}$ et al. Apoptosis and delayed degeneration after spinal cord injury in the rats and monkeys. Nature Med 1997; 3: $73-76$ 